\title{
Synapsine I et transmission de l'influx nerveux
}

Le couplage entre la dépolarisation de la membrane présynaptique et la libération des neurotransmetteurs lors du passage de l'influx nerveux est récemment devenu accessible à l'expérimentation. La dépolarisation provoque l'entrée du calcium dans les terminaisons nerveuses, entrée qui déclenche le passage à l'extérieur du contenu de vésicules appelées vésicules synaptiques. La recherche des mécanismes moléculaires qui sous-tendent la transmission synaptique à partir des cellules nerveuses s'est orientée dans deux directions principales : la caractérisation de protéines spécifiques des neurones et l'étude du rôle de la phosphorylation des protéines dans la régulation de la transmission. Ces deux voies ont convergé vers une même protéine, la synapsine $\mathrm{I}$.

Découverte en 1977 par Greengard et son équipe (Yale puis New York) et appelée d'abord protéine I [1], la synapsine est localisée exclusivement dans les neurones, plus précisément à la surface cytoplasmique des vésicules synaptiques, avec lesquelles elle contracte des liaisons. C'est une protéine très basique, de $\mathrm{pH}$ isoélectrique voisin de 10. Elle comporte [2] un domaine globulaire ("tête") et une " queue " allongée. Un ADN complémentaire obtenu à partir de cerveau de rat, a été cloné et séquencé, et a permis de déduire la séquence d'une protéine de 691 acides aminés pour une taille de $73 \mathrm{kD}$ [3] et d'interpréter certaines propriétés de la synapsine. La principale de ces propriétés est que la synapsine est phosphorylable par des enzymes capables d'intervenir dans la transmission synaptique. Deux systèmes ont été plus particulièrement étudiés : une protéine kinase AMPc dépendante agissant uniquement sur la " tête " terminale, et une protéine kinase

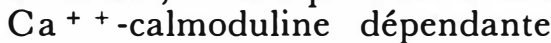
agissant sur la partie C-terminale ; ces derniers sites de phosphorylation sont proches de trois régions à séquence répétitive qui sont de bons candidats comme sites de liaison aux ligands, notamment aux vésicules synaptiques.

La synapsine interagit en effet avec toute une série de constituants tels que la spectrine cérébrale (ou foldine) ainsi qu'avec trois. classes de filaments cytoplasmiques : microtubules, neurofilaments [4] et actine $F$ (forme fibrillaire). L'interaction est forte quand la synapsine est déphosphorylée, et elle est très diminuée par la phosphorylation. C'est dans le cas de l'actine que les mécanismes ont été examinés le plus à fond [5]. In vitro, la déphosphosynapsine est capable de "botteler " (bundle) l'actine F, comme si celleci se trouvait condensée en bottes, ou gerbes, apparemment enserrées dans un lien de synapsine; la formation d'une botte met en jeu environ 10 molécules d'actine pour une de synapsine ; il est probable que la synapsine possède plusieurs sites de liaison avec l'actine. L'action de la synapsine est très diminuée si la tête est seule phosphorylée et elle est complètement abolie si tête et queue le sont toutes deux.

D'autres protéines ont des propriétés analogues : par exemple la villine de l'intestin, qui présente avec la synapsine une homologie de $50 \%$; et surtout la protéine dite 4,1 de la membrane du globule rouge dont la synapsine serait un analogue cérébral. La protéine 4,1 n'agit pas directement sur l'actine, mais forme un complexe ternaire avec l'actine et la spectrine, dont l'affinité est fortement réduite par la phosphorylation.

In vivo, les arguments les plus probants sont tirés de travaux sur la synapse géante du ganglion étoilé du calmar (Loligo pealii) [6]. Il est en effet possible de tester directement la libération des neurotransmetteurs en injectant dans la synapse un agent actif; la déphosphosynapsine diminue l'intensité du potentiel postsynaptique, mesure des neurotransmetteurs libérés, alors que l'injection de protéine kinase $\mathrm{Ca}^{+}+$-calmoduline dépendante l'augmente. Il n'est pas encore possible de décrire en détail les modalités de la transmission synaptique, mais des lignes directrices se dégagent [7]. Au repos, les vésicules synaptiques, liées à la synapsine I, sont retenues et leur exocytose n'est pas possible. Au cours de la dépolarisation de la membrane présynaptique, l'entrée de calcium stimule l'action de la protéine kinase $\mathrm{Ca}^{++}$-calmoduline dépendante, la synapsine est phosphorylée, donc inactivée, et les vésicules synaptiques peuvent livrer leur contenu à l'extérieur.

J.-C. D.

1. Ueda T, Greengard P. Adenosine 3' : 5' monophosphate-regulated phosphoprotein system of neuronal membranes. $J$ Biol Chem 1977 ; 252 : 5155-63.

2. Schiebler W, Jahn R, Doucet JP, Rothlein $J$, Greengard P. Characterization of synapsin I binding to small synaptic vesicles. $J$ Biol Chem 1986 ; 261 : 8383-90.

3. McCaffery CA, De Gennaro LJ. Determination and analysis of the primary structure of the nerve terminal specific phosphoprotein, synapsin I. EMBO J $1986 ; 5: 3167-73$.

4. Steiner JP, Ling E, Bennett V. Nearest neighbor analysis for brain synapsin I. $J$ Biol Chem 1987; 262 : 905-32.

5. Bähler M, Greengard P. Synapsin I bundles $\mathrm{F}$-actin in a phosphorylation-dependent manner. Nature 1987 ; 326 : 704-7.

6. Llinas R, McGuinness TL, Leonard CS, Sugimori M, Greengard P. Intraterminal injection of synapsin I or calcium-calmodulindépendent protein kinase II alters neurotransmitter release at the squid giant synapse. Proc Natl Acad Sci USA 1985; 82 : 3035-9.

7. Baines AJ. Synapsin I and the cytoskeleton. Nature $1987 ; 326: 646$. 